\title{
NF-кB p100 limits TNF-induced bone resorption in mice by a TRAF3-dependent mechanism
}

\author{
Zhenqiang Yao, Lianping Xing, and Brendan F. Boyce
}

Department of Pathology and Laboratory Medicine, University of Rochester Medical Center, Rochester, New York, USA.

\begin{abstract}
TNF and RANKL mediate bone destruction in common bone diseases, including osteoarthritis and RA. They activate NF- $\kappa B$ canonical signaling directly in osteoclast precursors (OCPs) to induce osteoclast formation in vitro. However, unlike RANKL, TNF does not activate the alternative NF- $\kappa B$ pathway efficiently to process the $\mathrm{I} \kappa \mathrm{B}$ protein NF- $\kappa \mathrm{B}$ p100 to NF- $\kappa \mathrm{B}$ p52, nor does it appear to induce osteoclast formation in vivo in the absence of RANKL. Here, we show that TNF limits RANKL- and TNF-induced osteoclast formation in vitro and in vivo by increasing NF- $K B$ p100 protein accumulation in OCPs. In contrast, TNF induced robust osteoclast formation in vivo in mice lacking RANKL or RANK when the mice also lacked NF- $\kappa B$ p100, and TNF-Tg mice lacking NF- $\kappa B$ p100 had more severe joint erosion and inflammation than did TNF-Tg littermates. TNF, but not RANKL, increased OCP expression of TNF receptor-associated factor 3 (TRAF3), an adapter protein that regulates NF- $\kappa B$ p100 levels in B cells. TRAF3 siRNA prevented TNF-induced NF- $\kappa B$ p100 accumulation and inhibition of osteoclastogenesis. These findings suggest that upregulation of TRAF3 or NF- $\kappa B$ p100 expression or inhibition of NF- $\kappa B$ P100 degradation in OCPs could limit bone destruction and inflammation-induced bone loss in common bone diseases.
\end{abstract}

\section{Introduction}

Osteoclasts, the cells that degrade bone, play a central role in bone destruction in common erosive bone diseases, including postmenopausal osteoporosis, RA, and periodontitis (1). In these conditions there is increased production of the proinflammatory cytokines TNF (2) and RANKL (3), members of the TNF superfamily (4) that stimulate bone resorption by osteoclasts $(5,6)$. Signaling through their receptors is mediated by recruitment of TNF receptor-associated factors (TRAFs) (7-9) and leads to activation of transcription factors including NF-кB, c-Fos, and NFATc1 $(10,11)$. Among the TRAF family of proteins, TRAF6 is essential for RANKL-induced osteoclast differentiation in vitro but not in vivo $(12,13)$, while TRAF2 may be required for TNF-induced osteoclast formation (14). TRAF5 is involved in both RANKL- and TNF-induced osteoclast formation (15). Similar to RANKL, but not many other osteoclast-stimulating factors, TNF can induce osteoclast formation directly from osteoclast precursors (OCPs) in vitro in the presence of M-CSF independent of RANKL/RANK signaling (16-18), by activation of NF-кB $(10,11)$. TNF induces fewer osteoclasts from WT OCPs and less bone resorption in vitro than RANKL $(11,17)$, but the molecular basis for this difference is unknown.

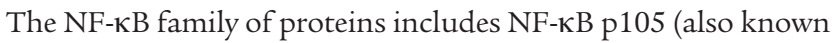

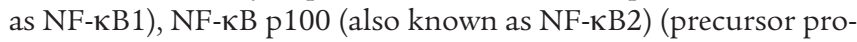
teins, which can act as inhibitory $\kappa \mathrm{B}$ proteins by binding to other NF-кB proteins), RelA (p65), RelB, and c-Rel $(19,20)$. In response to RANKL and TNF, p105 is processed constitutively to p50 (19-21), which forms dimers, typically with RelA $(19,20)$. RANKL and TNF

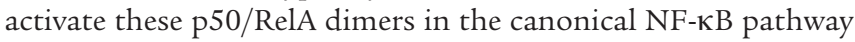
to promote OCP differentiation, but they also induce expression of p100 in these cells (22). p100 undergoes efficient proteasomal processing to $\mathrm{p} 52$ in response to RANKL through activation of

Conflict of interest: The authors have declared that no conflict of interest exists. Citation for this article: J. Clin. Invest. 119:3024-3034 (2009). doi:10.1172/JCI38716.
NF- $\kappa \mathrm{B}$-inducing kinase (NIK) and IKK $\alpha$ in the alternative pathway (23), thus releasing p52/RelB complexes to translocate to nuclei. In mice lacking NIK, unprocessed p100 can accumulate in OCPs and limit pathologic, but not basal, osteoclastogenesis induced by RANKL (23). TNF increases p100 protein levels slightly in OCPs (24), but it is not known whether TNF limits osteoclastogenesis through this mechanism. NIK is also involved in canonical NF-кB signaling (25). Its activity is regulated negatively by constitutive proteasomal degradation as a result of its association with TRAF3 (26-28), which limits $\mathrm{p} 100$ processing and inhibits noncanonical $\mathrm{NF}-\kappa \mathrm{B}$ signaling. Thus, ablation of TRAF3 protein in B cells in which CD40 or BAFF-R activation induces TRAF3 degradation and prevents NIK degradation, resulting in progressive accumulation of NIK and activation of the noncanonical NF- $\kappa B$ pathway through processing of p100 (26-28). Further, early postnatal lethality occurs in TRAF $3^{-1-}$ mice because of uncontrolled NIK activity; this is rescued by crossing the mice with $N f k b 2^{-/-}$mice (28). However, it is not known whether TNF affects TRAF3 or NIK levels in OCPs as a mechanism to control osteoclast numbers or activity.

Basal osteoclast formation requires expression of RANKL (29), RANK (30), and NF-KB p105 and p100 (31, 32), but not of TNF, TNF receptors (p55 and p75) (33), or TRAF2, -5 , or $-6(12,14,15$, $18)$, although the precise roles of TRAF2, -5 , and -6 in osteoclasts are controversial $(12,14,15,18)$. Despite compelling evidence for a strong proresorptive function of TNF, which induces osteoclast formation from $\mathrm{Rankl}^{-/-}$and $\mathrm{Rank}^{-/-}$OCPs in vitro when costimulated with TGF- $\beta(17,18)$, TNF does not induce osteoclast formation in vivo when administered to $\mathrm{Rank}^{-/-}$mice (34). These findings have led to the conclusion that RANKL/RANK signaling is a prerequisite for osteoclast differentiation in vivo (35). Here we report an unexpected role for TNF to limit TNF- and RANKLinduced osteoclastogenesis by inducing expression of NF- $\mathrm{\kappa B}$ p100 and TRAF3 in OCPs. TNF also induces robust osteoclast formation in vivo in $\mathrm{Rankl}^{-/-}$and $\mathrm{Rank}^{-/-}$mice when they also lack NF-кB 


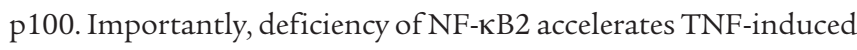
bone loss and joint inflammation in a model of RA, pointing to a role for NF- $\mathrm{KB}$ p100 to limit bone resorption and inflammation in diseases in which TNF expression is increased.

\section{Results}

$T N F$ induces expression of noncanonical $N F-\kappa B$ proteins differently from RANKL in OCPs. To investigate the possibility that TNF induces fewer osteoclasts from OCPs than RANKL by promoting accumulation of the inhibitory NF- $\mathrm{BB}$ p100 protein, we examined the expression pattern of p100 in WT OCPs. Both RANKL and TNF increased $p 100 / p 52$ mRNA levels within 4 hours of treatment by 11- and 7-fold, respectively, and these remained elevated by 7 - and 5 -fold, respectively, at 8 hours (data not shown). We examined p100 and p52 protein expression levels during our typical culture period (1-96 hours). TNF induced sustained accumulation of p100 between 4 and 72 hours (Figure 1A). In contrast, RANKL more efficiently processed p100 to p52 during this period (Figure $1 \mathrm{~A})$, and this was associated with the formation of larger numbers of osteoclasts (Figure 1B). p100 levels in TNF-treated cells were not different from those of PBS-treated controls at 96 hours, by which time osteoclast formation has peaked in these cultures (11). TNF also induced increased p52 levels, but to a lesser extent than RANKL between 8 and 48 hours, confirming that p100 is processed in response to TNF (24), especially at 72 and 96 hours, when TNF induces peak osteoclast formation (11). Both TNF and RANKL markedly increased RelB levels, but the effect of TNF was greater than that of RANKL from 48 to 96 hours (Figure 1A). Deficiency of p100 did not affect either TNF- or RANKL-mediated nuclear translocation of RelB (Supplemental Figure 1A; supplemental material available online with this article; doi:10.1172/ JCI38716DS1), presumably because RelB also can associate with p50 and translocate into nuclei (36). In contrast, TNF and RANKL overall had similar stimulatory effects on the expression levels of the canonical NF- $\kappa \mathrm{B}$ p50 and $\mathrm{p} 65$ proteins during osteoclastogenesis (24-96 hours), with some variation at each time point (Figure 1A). Of note, although both TNF and RANKL mediated rapid NF-кB p65 nuclear translocation, the effect of TNF was more sustained than that of RANKL (Supplemental Figure 1, B and C). We had observed this previously in OCPs from NF-кB1/2 doubleknockout mice that do not form osteoclasts (11).

TNF-induced NF-КB 100 limits osteoclastogenesis. To determine whether TNF-induced NF-кB p100 limits the number of osteoclasts formed in response to TNF, we treated $N f k b 2^{-1-}$ and WT OCPs with TNF and found significantly more osteoclasts from $N f k b 2^{-/-}$than from WT cells, the numbers being similar to those

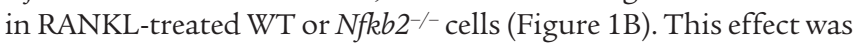
not associated with induction in vitro or in vivo in OCPs of IFN- $\beta$ (data not shown), which limits RANKL-induced osteoclastogenesis by downregulating c-Fos expression in OCPs (37). Importantly, osteoclasts induced by TNF from $N f k b 2^{-/-}$OCPs formed resorption pits as effectively as those induced by RANKL (Figure 1B). TNF activates $c$-Fos and NFATc1, the same critical transcriptional factors activated by RANKL to induce osteoclast differentiation, but TNF induces these to a lesser extent than RANKL when mature osteoclasts are formed (11). However, we found that TNF induced a fold induction of c-Fos and NFATc1 similar to that of RANKL when osteoclasts were forming in $\mathrm{Nfkb}^{-\gamma_{-}}$cells (data not shown). Erk and p38 signaling are also involved in osteoclast differentiation and are activated in response to RANKL and TNF (38). We found that TNF induced stronger activation of both Erk and p38 than RANKL, and this was observed in both WT and Nfkb2-/- cells (data not shown).

Nfkb2- $2^{--}$OCPs lack both $p 100$ and $p 52$. To determine whether p100 or p52 is responsible for the inhibitory effect of TNF, we infected $N f k b 2^{-/-}$and WT OCPs with $p 100$, $p 52$, or GFP control retrovirus and treated them with TNF. p100 inhibited TNF- and RANKLinduced osteoclastogenesis significantly in both $\mathrm{Nfkb2}^{-/-}$and WT OCPs (Figure 1C). p52 also caused a small but significant reduction in osteoclast numbers (Figure 1C), which may be due to a minor inhibitory effect of p52 homodimers, but this effect was much less than that of $\mathrm{p} 100$. High expression of these retrovirally induced proteins was confirmed in infected WT OCPs (Figure 1C).

To determine whether $\mathrm{p} 100$ limits TNF-induced osteoclastogenesis in vivo, we injected TNF or PBS vehicle into the supra-calvarial subcutaneous tissues of $\mathrm{Nfkb2}^{-/-}$and $\mathrm{Nfkb2}^{+/-}$control mice twice daily for 5 days and examined its effects on osteoclastogenesis and resorption. Basal osteoclast numbers as well as osteoclast surface

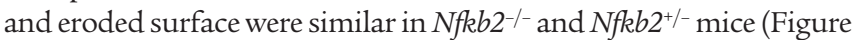
1D). Mean values for these parameters of bone resorption increased

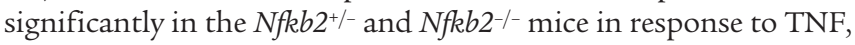

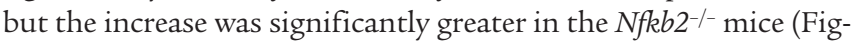
ure 1D). Serum levels of tartrate-resistant acid phosphatase $5 \mathrm{~b}$ (TRAP5b), a specific marker of bone resorption released by osteoclasts, were significantly higher in $\mathrm{Nfkb}^{-/-}$mice than in control mice treated with TNF $(7.3 \pm 0.5$ vs. $5.4 \pm 0.8 \mathrm{U} / 1 ; P<0.01)$, confirming that $N f k b 2$ deficiency enhances TNF-induced bone resorption. We

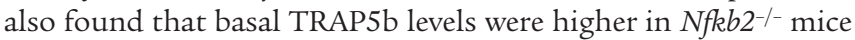
than in their littermate controls $(6.2 \pm 0.1$ vs $3.6 \pm 0.4 \mathrm{U} / 1 ; P<0.01)$,

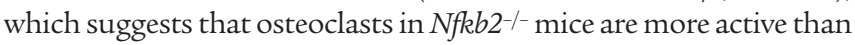
WT osteoclasts, as we did not observe any difference in the number of osteoclasts at this time point. This effect differs from those we reported previously with IL-1, which induced similarly increased

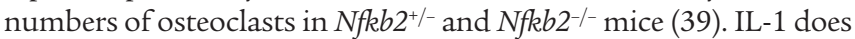
not induce osteoclast formation from WT OCPs in the absence of RANKL but can do so when the cells overexpress c-Fos, which is activated by, and downstream of, NF-кB (40).

TNF induces osteoclastogenesis in Rank ${ }^{-/-}$or Rankl $l^{-/}$mice in the absence

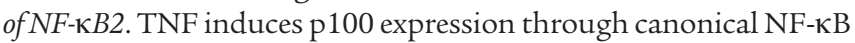
signaling (41). To test the hypothesis that $\mathrm{p} 100$ induced by TNF prevents osteoclast formation in vivo in $\mathrm{Rank}^{-/-}$mice, we first examined whether TNF increases the expression of NF- $\kappa \mathrm{B}$ p100 protein in Rank $^{-/-}$or Rankl $l^{-/}$OCPs. Similar to its effects in WT cells, TNF induced $\mathrm{p} 100$ accumulation but not $\mathrm{p} 52$ protein in Rank $^{-/-}$and Rankl $^{-/-}$cells (Figure 2A), indicating that TNF-induced p100 protein expression is independent of RANK signaling. There was some variability in basal levels of p100 expression from one experiment to another, which is a recognized feature of currently available antibodies to this protein, but basal levels of expression overall did not vary significantly among WT, Rank-/-, and Rankl $\mathrm{I}^{-/}$ OCPs. As expected, RANKL did not have any effect on p100/p52 protein in Rank $^{-/-}$cells, while it clearly increased p52 in Rankl-/cells (Figure 2A). Of note, different from WT cells, RANKL did not increase p100 levels in $\mathrm{Rankl}^{-/-}$OCPs, possibly because they had not encountered RANKL previously and therefore might be more sensitive to RANKL and completely process p100 to p52.

We next generated Rank $k^{-/-} / \mathrm{Nfkb2}^{-/-}$and $\mathrm{Rankl}^{-/-} / \mathrm{Nfkb}^{-/-}$mice to determine whether TNF could induce osteoclastogenesis in the absence of NF-кB2 and either RANK or RANKL. First, we treated

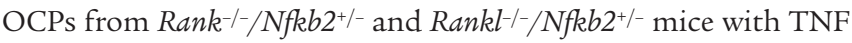


A

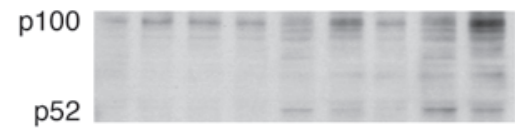

RelB $=-0-000$

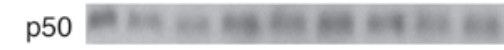

p65

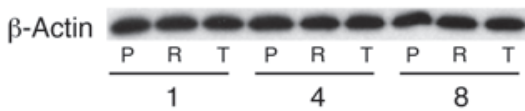

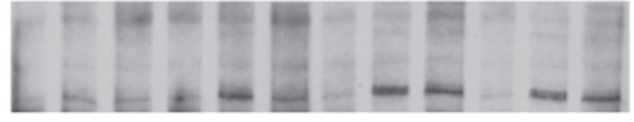

$+5-60-\infty-\infty$

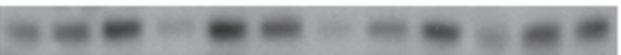

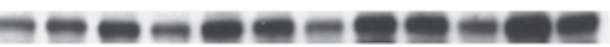

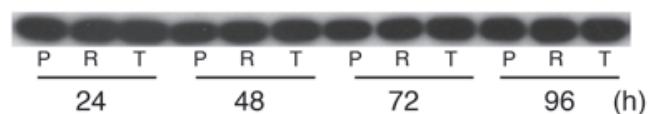

B

3

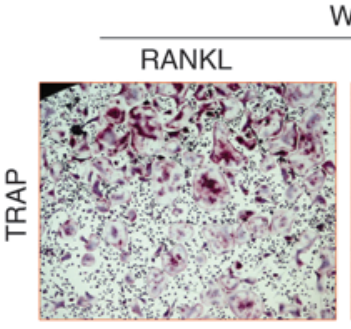

\section{WT}

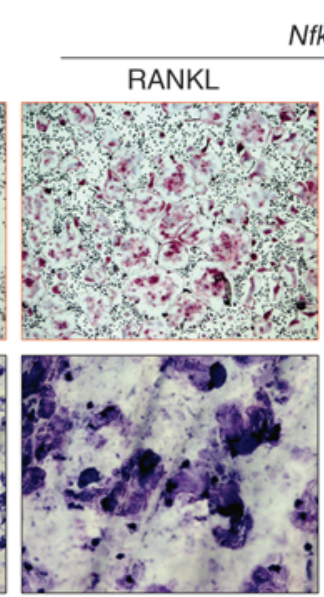

Nfkb2--
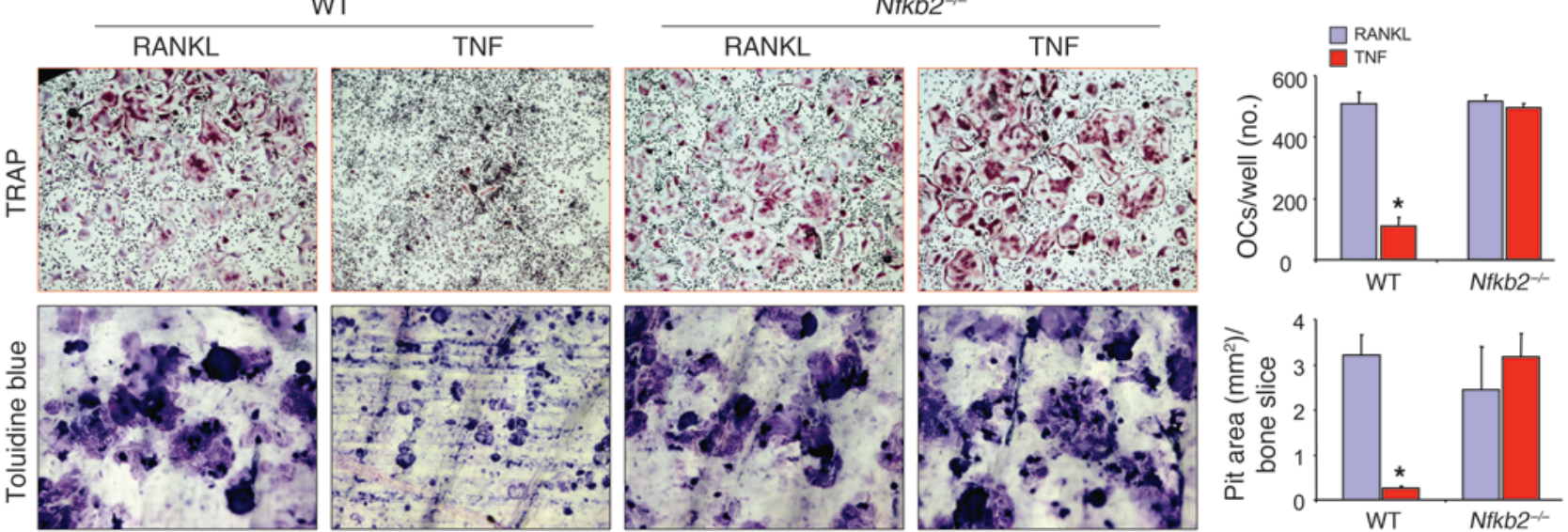

C
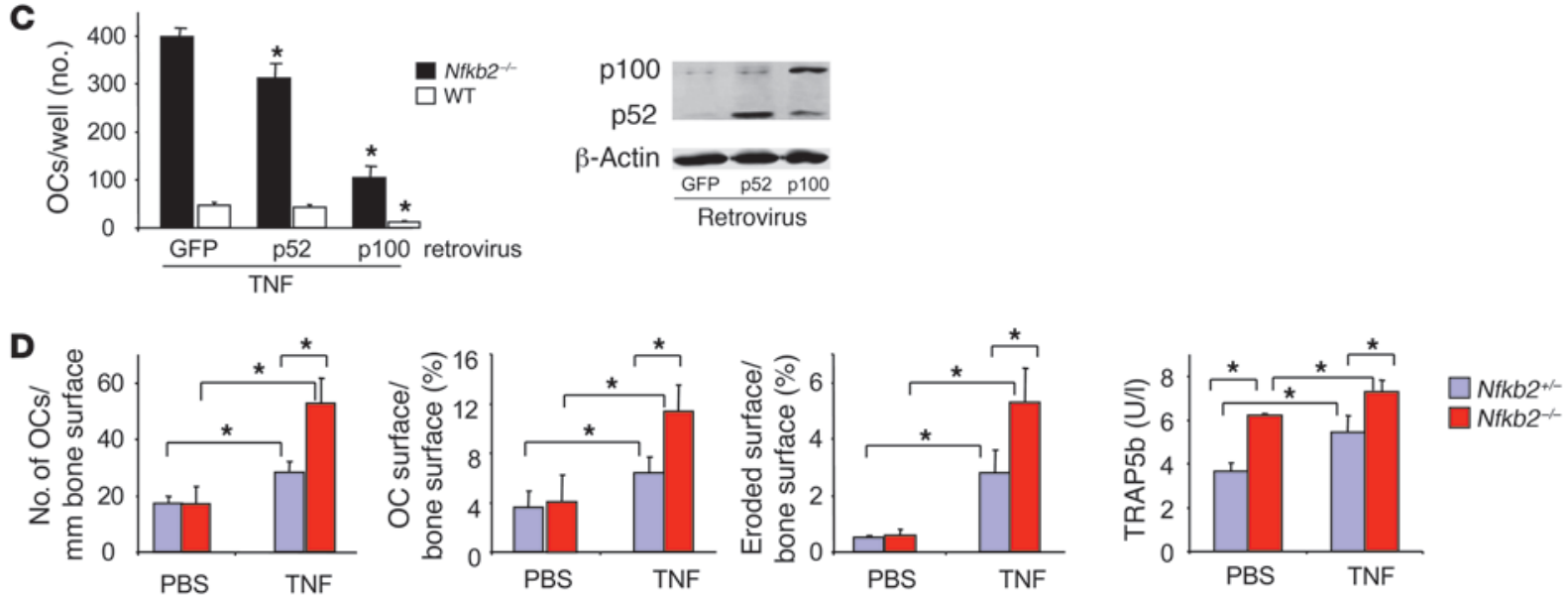

Figure 1

TNF-induced expression of NF-кB p100 inhibits osteoclastogenesis. (A) WT mouse OCPs, cultured from splenocytes with M-CSF for 3 days, were treated with RANKL or TNF for the indicated times. NF-KB proteins in whole-cell lysates were determined by Western blot. Experiments were repeated at least twice with similar results. P, PBS; R, RANKL $10 \mathrm{ng} / \mathrm{ml}$; T, TNF $20 \mathrm{ng} / \mathrm{ml}$. (B) WT or Nfkb2 $2^{-/-}$OCPs were treated with RANKL or TNF directly on plastic or bone slices in 96-well plates in the presence of M-CSF for 2 and 5 days, respectively, to induce osteoclasts (OCs) and resorption pits. Top: Representative TRAP-stained osteoclasts (original magnification, $\times 4$ ) and toluidine blue-stained pits (original magnification, $\times 20)$. Bottom: Osteoclast number and resorption pit area ( $n=4 /$ group; ${ }^{\star} P<0.05$ vs RANKL). (C) Nfkb2 ${ }^{-/-}$or WT OCPs were infected with GFP, p100, or p52 retroviruses for 2 days and treated with TNF for 2 more days. Osteoclast numbers were counted (left panel; ${ }^{*} P<0.05$ versus GFP), and the infection efficiency was confirmed by Western blot from the infected WT OCPs (right panels). (D) Murine TNF $\left(0.5 \mu \mathrm{g}\right.$ in $10 \mu \mathrm{l}$ PBS) or $10 \mu \mathrm{l}$ PBS were injected twice daily over the calvariae of 4-week-old $\mathrm{Nfkb2}^{-/-}$or $\mathrm{Nfkb2}^{+/-}$control mice for 5 days ( $n=4$ /group). The number of osteoclasts/mm bone surface, percentage of osteoclast surface/bone surface, and percentage of eroded surface/ bone surface were measured in TRAP-stained calvarial bone sections, and serum TRAP5b was tested with ELISA.

and found that they formed slightly but significantly more osteoclasts than did OCPs from $N f k b 2^{+-}$littermates (Figure 2B). This likely reflects the fact that Rank $^{-/}$(42) and Rankl-/ (data not shown) mouse spleens contain more OCPs than do spleens of WT mice because of extramedullary hematopoiesis that results from their lack of an adequate marrow cavity. OCPs from $\mathrm{Rank}^{-/} / \mathrm{Nfkb2^{-/ }}$ and Rankl ${ }^{-/} / \mathrm{NfRb}^{-/-}$mice treated with TNF formed significantly more osteoclasts than did OCPs from Rank $k^{-/} / \mathrm{Nfkb}^{+/-}$and Rankl-// 
A

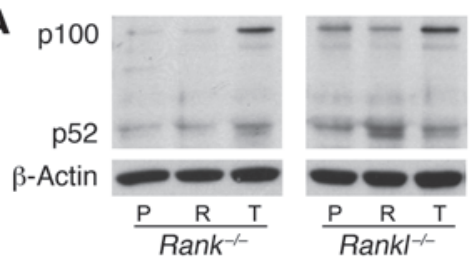

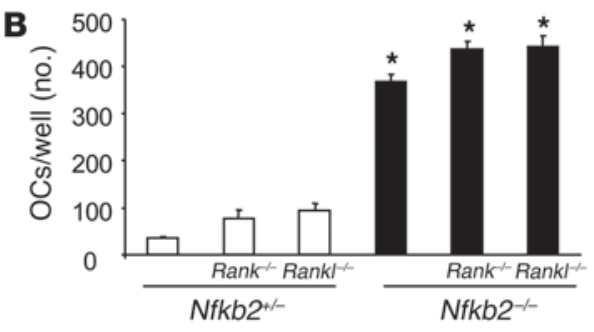

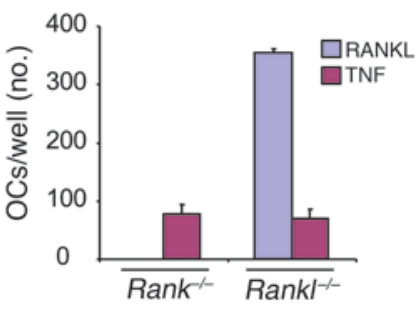

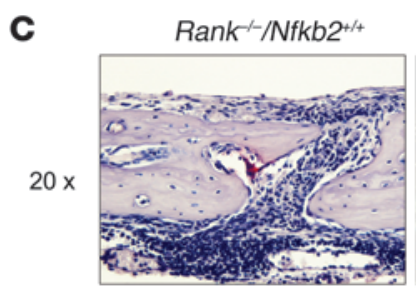

Rank/Nfkb2 dKO

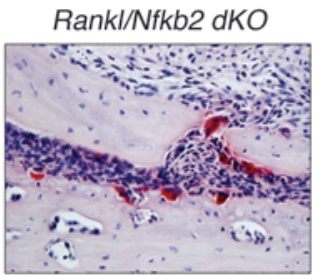

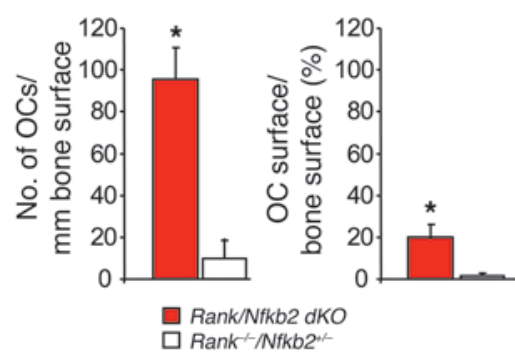

D

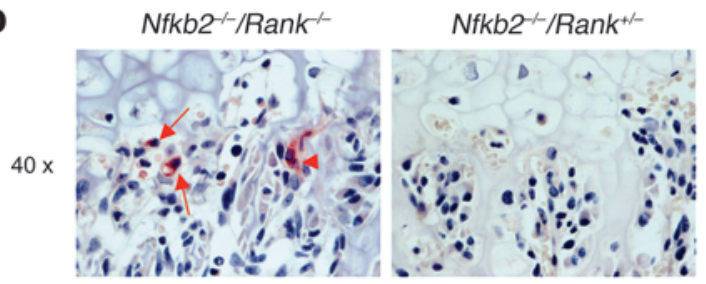

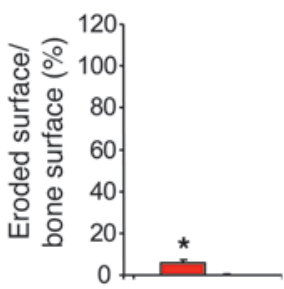

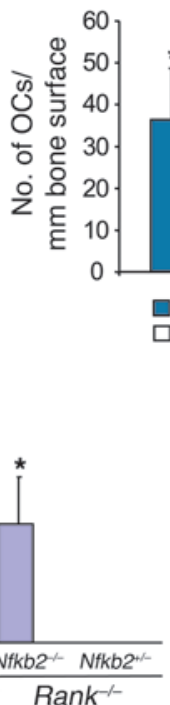

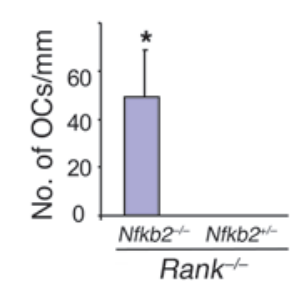

\section{E}

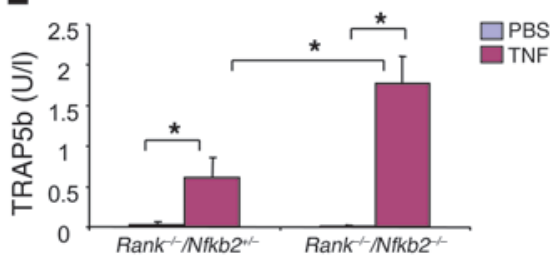

Figure 2

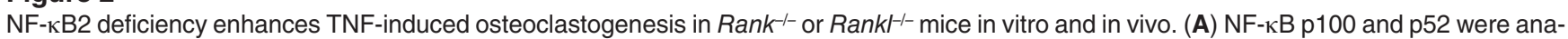
lyzed by Western blot in whole-cell lysates of PBS-, RANKL-, or TNF-treated (8 hours) OCPs from Rank-1- or Rankl ${ }^{-1-}$ mice. (B) Left: OCPs from

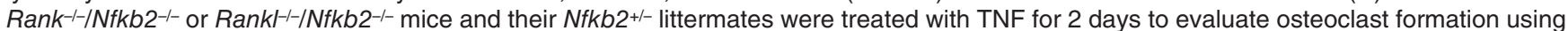

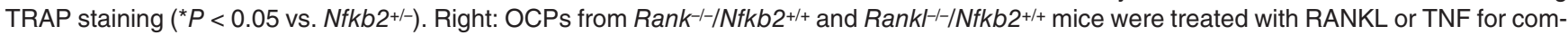

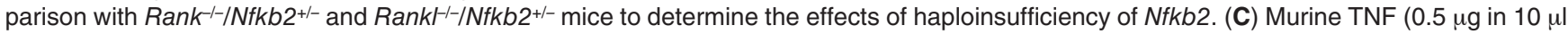

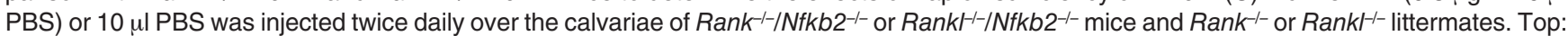
TRAP-stained sections show numerous actively resorbing TRAP+ osteoclasts locally in calvarial sections (original magnification, $\times 20$ ) from TNFinjected Rank $\mathrm{k}^{-1 /} / \mathrm{Nfkb}^{-/-}$or Rankl/-/Nfkb2-/- mice. Bottom: Numbers and surface extent of osteoclasts $(n=3 /$ genotype). Occasional osteoclasts

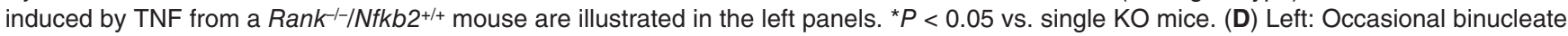
(arrowhead), but mainly mononuclear (arrows), TRAP+ cells (left panel) formed beneath hypertrophic chondrocytes in the growth plate of the

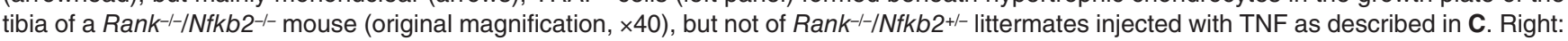
Osteoclast numbers (expressed per mm of length of growth plate) counted in representative sections. (E) Serum TRAP5b levels were tested

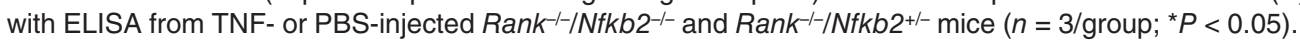

$\mathrm{Nfkb2} 2^{+/-}$mice, the numbers being slightly higher than those from $\mathrm{Nfkb2}^{-/-}$OCPs (Figure 2B). We also found that TNF induced the formation of bone-resorbing osteoclasts from $\mathrm{Rank}^{-/-} / \mathrm{Nfkb2^{+/- }}$ and $\mathrm{Rankl}^{-/-} / \mathrm{Nfkb2}^{+/-}$OCPs without the addition of TGF- $\beta$, which was suggested to be necessary as a pretreatment (18). A neutralizing TGF- $\beta$ antibody did not prevent these effects, nor did the addition of TGF- $\beta$ increase TNF-induced osteoclast numbers (data not shown). To exclude the possibility that haploinsufficiency of $N f k b 2$ affects the status of Rank $^{-/-}$and Rankl $^{-/-}$osteoclast differentiation, we treated spleen cells from Rank $^{-/-}$and Rankl $^{-/-}$mice with TNF in the presence of M-CSF and found that similar osteoclast numbers were formed as with OCPs from $\mathrm{Rank}^{-/-}$and $\mathrm{Rankl}^{-/-}$mice with $\mathrm{Nfkb2}$ haploinsufficiency (Figure 2B, right panel). We believe that we were able to induce osteoclastogenesis in our cultures because we used lower numbers of M-CSF-dependent OCPs from these KO mice than we used from WT controls. The KO mice had higher numbers of OCPs in their spleens, as described above, and when we used similar numbers of OCPs from Rankl-/- or Rank ${ }^{-/-}$mice as from WT mice, 
A

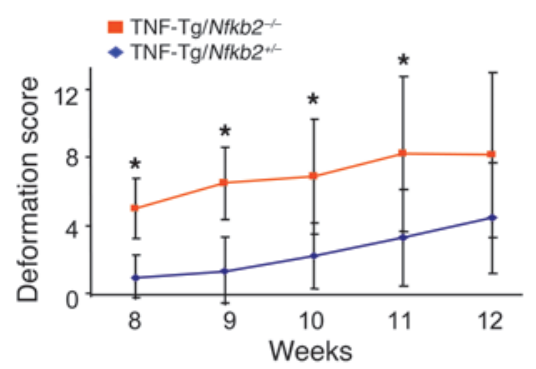

B

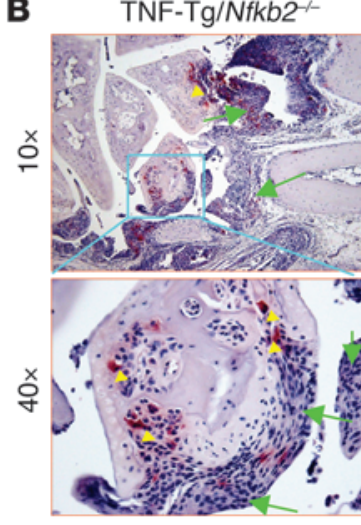

TNF-Tg/Nfkb2 $2^{+/}$

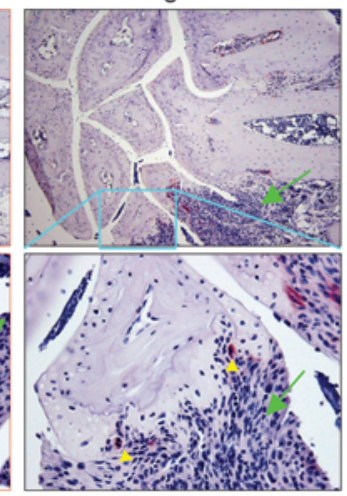

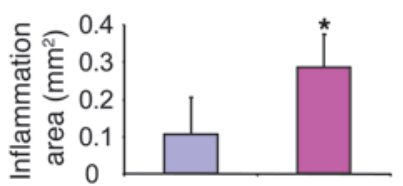

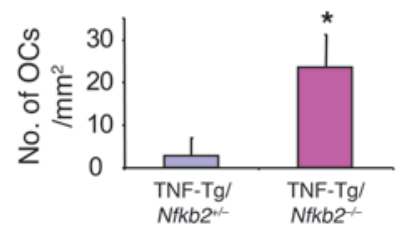

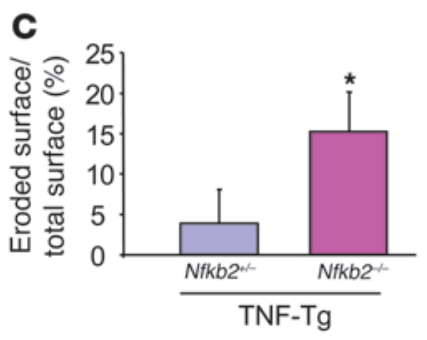

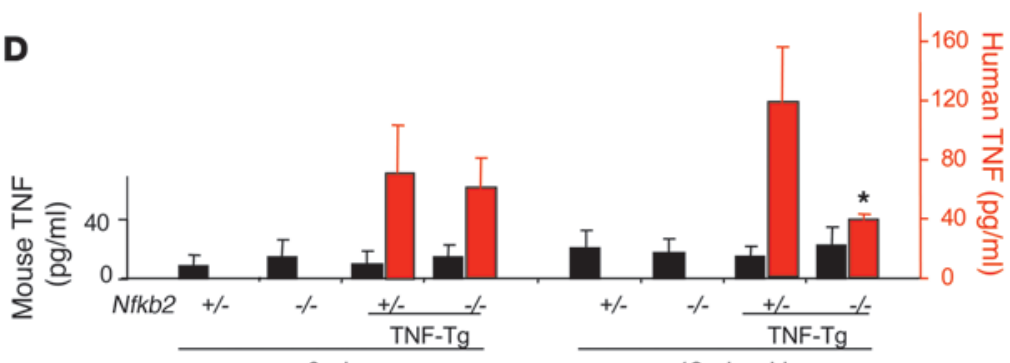

6 wks

12 wks old

\section{Figure 3}

Increased joint inflammation and osteoclastogenesis in TNF-Tg/Nfkb2-/- mice. (A) Age-related changes in clinically assessed joint deformation scores showed that joint deformation occurred earlier in the TNF-Tg/Nfkb2--- mice $(n=7)$ than in their TNF-Tg/Nfkb2 $2^{+-}$littermates $(n=8)$. (B) Representative TRAP-stained sections (original magnification, $\times 20$ ) from 12-week-old animals showed more severe wrist joint inflammation (green arrows) and more osteoclasts (yellow arrowheads) in a TNF-Tg/Nfkb2-/- mouse than in a TNF-Tg/Nfkb2 $2^{+/-}$mouse. Histomorphometric analysis showed that the area of inflammatory tissue (upper panel) and osteoclast numbers (lower panel) were increased in the wrists of TNF-Tg/ $N f k b 2^{-1-}$ mice. ${ }^{*} P<0.05$. (C) The percentage of cartilage eroded surface/total joint surface was measured in carpal bones of 6-week-old mice ( $n=5 /$ group). (D) Serum levels of murine TNF (black bars) and human TNF (red bars) were tested with ELISA at 6 and 12 weeks of age $\left({ }^{\star} P<0.05\right.$ vs. TNF-Tg/Nfkb+/- littermates).

TNF did not induce osteoclastogenesis (data not shown), presumably because their increased density inhibits differentiation.

Although absence of $N f k b 2$ itself did not induce any osteoclasts in Rank $^{-/-}$or Rankl $l^{-/-}$mice (PBS injection; data not shown), TNF induced many osteoclasts and resorption lacunae in the calvarial bones of the Rank ${ }^{-/-} / \mathrm{Nfkb2}^{-/-}$and $\mathrm{Rankl}^{-/-} / \mathrm{Nfkb2}^{-/-}$mice following local injection, associated with increased osteoclast and eroded surfaces (Figure 2, C and D). Only occasional osteoclasts were observed in the sections of TNF-injected Rankl-/- or Rank $k^{-/-}$mice, as reported previously (34). We also observed small numbers of TRAP ${ }^{+}$osteoclasts in the long bones of the TNF-injected Rank $k^{-/-} / \mathrm{fkbl}^{-{ }^{--}}$and

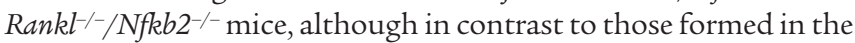
calvariae, these were mainly mononuclear cells located predominantly along the edge of the growth plates (Figure 2D) or in the centers of the physes. These cells had no effect on the increased bone volume in these osteopetrotic mice, presumably reflecting the short period of 5-day administration and their small size. To assess the possible function of these cells further, we measured serum TRAP5b levels. TRAP5b was undetectable in $\mathrm{Rank}^{-/-} / \mathrm{Nfkb2}^{+/-}$and $\mathrm{Rank}^{-/-} / \mathrm{Nfkb2-/-}$ mice and was slightly but significantly increased

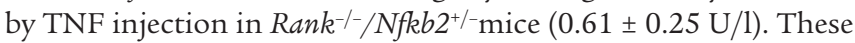

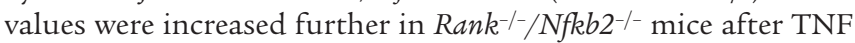
injection (1.77 $\pm 0.34 \mathrm{U} / \mathrm{l}$; Figure $2 \mathrm{E})$, confirming that osteoclasts induced by TNF in Rank $^{-/-}$mice are functional and that NF-кB2 deficiency enhances TNF-induced osteoclastogenesis and bone resorption in the mice lacking RANK signaling. TRAP5b was not observed in serum or osteoclasts in bone sections from vehicletreated $\mathrm{Rank}^{-/-} / \mathrm{Nfkb2}^{-/-}$or $\mathrm{Rankl}^{-/-} / \mathrm{Nfkb2}^{-/-}$mice, presumably because the concentration of endogenous TNF in the marrow cavities of these mice is low and not sufficiently high to induce OCP differentiation even in the absence of $\mathrm{p} 100$.

TNF-Tg mice lacking NF-КB 100 have more severe joint erosion and inflammation and systemic bone loss than TNF-Tg mice. To determine whether the absence of NF- $\mathrm{KB} 2$ would enhance joint erosion in TNF-Tg mice, we generated TNF-Tg/Nfkb2-/- mice and found that they developed joint deformity earlier than their TNF-Tg littermates, that is, at 8 weeks versus 12 weeks of age (Figure $3 \mathrm{~A}$ ). At 12 weeks of age, TNF-Tg/Nfkb2 $2^{-/}$mice had significantly increased areas of inflammation and osteoclast numbers in their forepaw joints assessed histomorphometrically (Figure 3B). However, we observed no significant difference in the types of inflammatory cells or in the appearance of the hyperplastic synoviocytes in the TNF-Tg/Nfkb2 $2^{-/}$mice compared with control mice upon histologic analysis. FACS analysis showed that $\mathrm{Nfkb2}^{-/-}$mice had significantly reduced numbers of $\mathrm{B} 220^{+} \mathrm{B}$ cells in their spleens and peripheral blood compared with $\mathrm{Nfkb}^{+/-}$control mice. This fea-

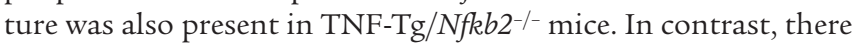
was no difference in numbers of $\mathrm{CD}^{+}, \mathrm{CD}^{+}$, or $\mathrm{CD} 8^{+}$cells among

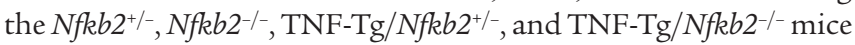
(data not shown). 

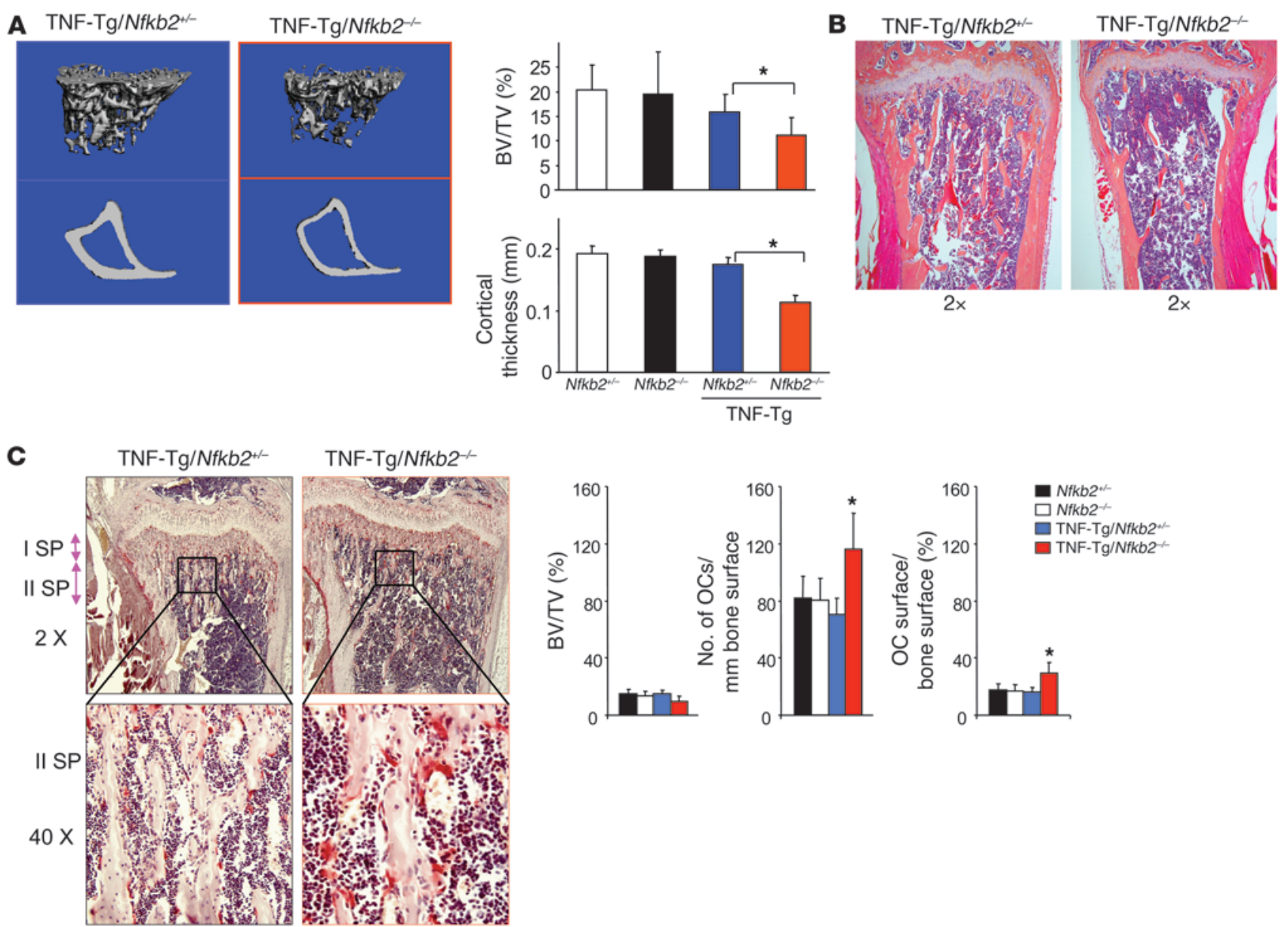

Figure 4

More severe systemic bone loss in TNF-Tg/Nfkb2-/- mice. (A) Tibiae from 12-week-old Nfkb ${ }^{+/-}(n=4), N f k b 2^{-/-}(n=4), \mathrm{TNF}-\mathrm{Tg} / \mathrm{Nfkb2}^{+/-}(n=7)$, and TNF-Tg/Nfkb2-/- mice $(n=8)$ were subjected to $\mu \mathrm{CT}$ scanning. Representative images (left) and data analysis (right) showed reduced trabecular bone volume and cortical bone thickness in TNF-Tg/Nfkb2-- mice. (B) H\&E-stained sections of tibiae (original magnification, $\times 2$ ) showed decreased trabecular bone in the metaphyseal regions and thinner cortices (green arrow) in TNF-Tg/Nfkb2 ${ }^{-l-}$ mice. (C) TRAP-stained sections of 6-week-old mice showed increased numbers of osteoclasts in the secondary spongiosa of the proximal tibia of a TNF-Tg/Nfkb2-l- mouse (left panels), which was confirmed by histomorphometric analysis (right panels). BV/TV, bone volume/tissue volume. ${ }^{*} P<0.05$ vs. TNF-Tg/Nfkb2 ${ }^{+/-}$ mice. Original magnification, $\times 2$ (top), $\times 40$ (bottom).

TNF-Tg/Nfkb2-/- mice also had reduced long bone trabecular bone volume and cortical thickness compared with TNF-Tg/Nfkb2+/- mice (Figure 4A), which we confirmed morphometrically by 3 -dimensional $\mu$ CT imaging (Figure 4B). These TNF-Tg/Nfkb2-/- mice had lost almost all of their metaphyseal trabecular bone (Figure 4A), making it difficult to quantify and normalize osteoclast parameters histomorphometrically. However, in 6-week-old TNF-Tg/Nfkb2-/- mice, which had slightly but not significantly lower trabecular bone volume/tissue volume ratios than TNF-Tg mice, metaphyseal osteoclast numbers and surfaces were increased significantly (Figure 4C). These mice also had enhanced erosion of cartilage on their carpal bone joint surfaces compared with TNF-Tg/Nfk $b 2^{+/-}$littermates (Figure 3C). Bone formation rates assessed in undecalcified bone sections following double calcein labeling were similar in the TNF-Tg/ $N f k b 2^{-1-}$ and control mice (data not shown).

We next determined whether NF-кB2 deficiency influences human $(\mathrm{Tg})$ or mouse serum TNF concentrations in these mice. Murine TNF values were similar among the groups in both 6- and 12-week- old mice. NF-кB2 deficienc0y did not affect human TNF concentrations in 6-week-old TNF-Tg mice, but mean values were significantly

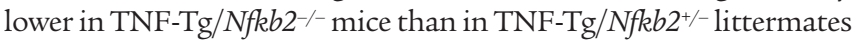
aged 12 weeks. We do not have an explanation for the reduction of TNF in these mice, but by this age TNF-Tg/Nfkb2-/-mice were much smaller than control mice, and some had already died. The cause of this early mortality requires further study.

TNF attenuates RANKL-induced osteoclastogenesis in vitro through $N F-\kappa B$ p100. Many cytokines, including RANKL, TNF, IL-1, and $\mathrm{M}-\mathrm{CSF}$, are involved in bone destruction in pathological conditions. Of these, RANKL and TNF can support the final stages of OCP differentiation to osteoclasts. Therefore, it is important to study how TNF and RANKL work together to control osteoclastogenesis. A previous study reported that TNF synergizes with RANKL to stimulate osteoclastogenesis in vitro (35) when both cytokines are added at the beginning of the culture period. However, the synergistic effect occurs only in RANKL pretreated cells (43), which we confirmed (data not shown). We then used a dif- 
A
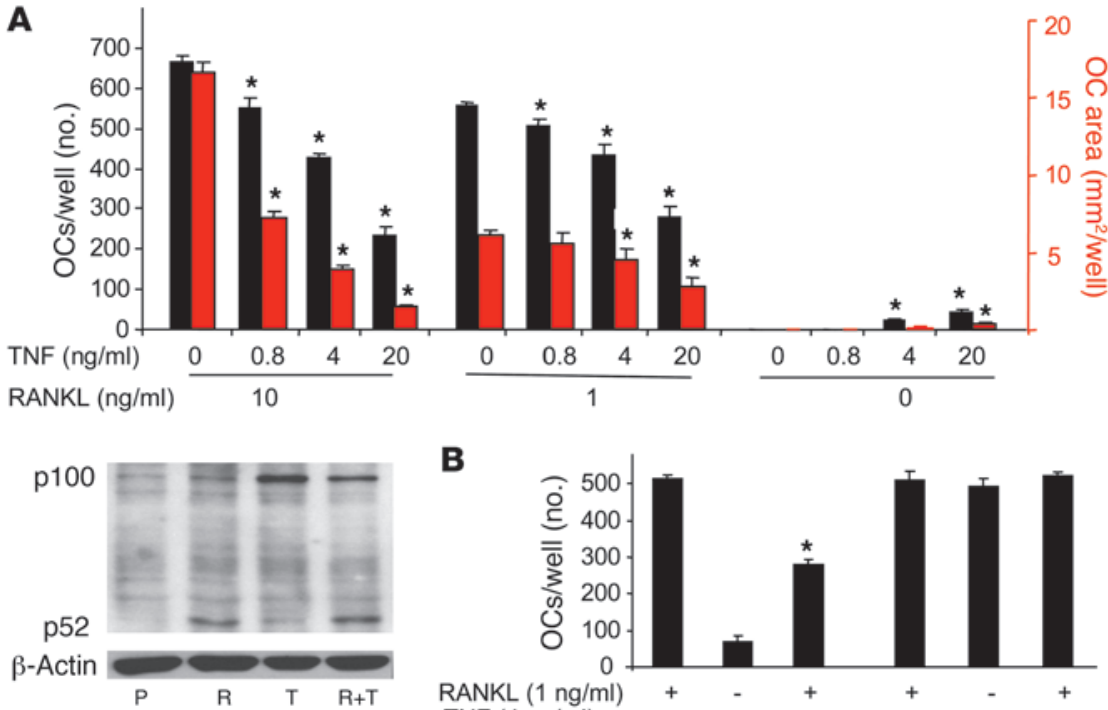

B

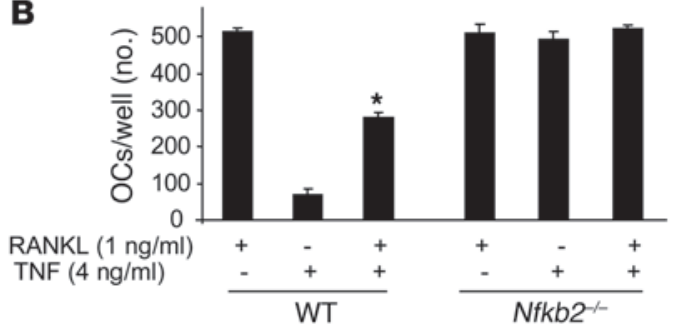

\section{Figure 5}

TNF-induced NF-KB p100 inhibits RANKLinduced osteoclastogenesis. (A) WT mouse spleen cells were cultured with M-CSF for 3 days, and RANKL and/or TNF were added at the indicated doses for 2 more days to generate osteoclasts. Top: TNF dose-dependently inhibited RANKL-induced osteoclastogenesis, assessed by osteoclast number (black bars) and area (red bars) per well. Bottom: The protein levels of NF-kB p100 and p52 were analyzed with Western blot and assessed in whole-cell lysates extracted from RANKL-treated (10 ng/ $\mathrm{ml})$ and/or TNF-treated (20 ng/ml) WT mouse OCPs at 8 hours. (B) The inhibitory effect of TNF on RANKL-induced osteoclastogenesis was abolished in $\mathrm{Nfkb2}^{-1-}$ OCPs $\left({ }^{*} P<0.05\right.$ vs. RANKL treatment alone; 4 wells/group). The same experiments were repeated at least twice with similar results. ferent osteoclastogenesis protocol in which spleen cells are first cultured with M-CSF for 3 days, to enrich for OCPs, and then TNF with or without RANKL was added to these cells for a further 2-3 days. TNF inhibited RANKL-induced osteoclastogenesis in a dose-dependent $(0.8-20 \mathrm{ng} / \mathrm{ml})$ manner (Figure 5A) at both optimal $(10 \mathrm{ng} / \mathrm{ml})$ and low $(1 \mathrm{ng} / \mathrm{ml})$ RANKL concentrations. Of note, although the number of osteoclasts induced by $1 \mathrm{ng} / \mathrm{ml}$ RANKL was just slightly less than the optimal dose, osteoclasts formed later and were smaller in these cultures, and this was evident as a reduced osteoclast area (Figure 5A). TNF alone (4 and $20 \mathrm{ng} / \mathrm{ml}$ ) induced small numbers of osteoclasts in the absence of RANKL, as expected. In these experiments, NF- $\mathrm{B}$ p100 levels in WT OCPs treated with TNF $(20 \mathrm{ng} / \mathrm{ml})$ plus RANKL $(10 \mathrm{ng} / \mathrm{ml})$ were significantly higher than in cells treated with RANKL alone, but the levels were less than those in cells treated with TNF alone, presumably because RANKL induced some proteasomal degradation of p100 through NIK, despite the inhibitory effect of TNF (Figure 5A). Accordingly, p52 expression was higher in the cultures of RANKL plus TNF than in those treated with TNF alone.

We next treated WT and Nfkb2-/- OCPs with low but effective doses of TNF with or without RANKL and found that TNF $(4 \mathrm{ng} / \mathrm{ml})$ significantly reduced osteoclastogenesis induced by RANKL $(1 \mathrm{ng} / \mathrm{ml})$

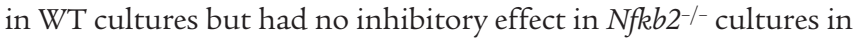
which both cytokines had nearly maximal osteoclastogenic effects either alone or in combination (Figure 5B), confirming that NF- $\mathrm{KB}$ p100 mediates this TNF-induced inhibition of osteoclastogenesis.

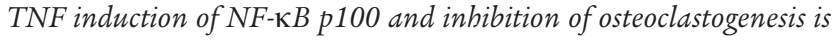
mediated by TRAF3. To determine the mechanism whereby TNFinduced NF-KB p100 accumulation limits osteoclastogenesis, we examined the effects of TNF and RANKL on the expression of TRAFs in OCPs. TRAFs directly interact with TNF superfamily receptors and trigger intracellular signaling events, including NIK-mediated processing of p100 to p52 (7). In addition, TRAF2 and -3 can also mediate proteasomal degradation of signaling molecules $(26,27)$. We found that neither RANKL nor TNF affected the mRNA expression levels of TRAF3 or - 6 (data not shown). However, both RANKL and TNF increased the protein levels of TRAF6, which activates NIK, and this is consistent with our observation that both cytokines slightly elevated NIK protein levels (Figure 6A). Importantly, TNF induced significantly higher levels of TRAF3, paralleling the elevated NF-אB p100 levels (Figure 6A). RANKL alone slightly reduced TRAF3, but it significantly decreased TNF-induced TRAF3 protein levels, which was matched by relatively lower levels of p100 (Figure 6A). Neither TNF nor RANKL affected protein levels of TRAF2 or -5 significantly in these cultures (data not shown).

To determine how TRAF3 is regulated by TNF, we added cycloheximide to the cultures of WT OCPs treated with PBS, RANKL, or TNF to prevent the synthesis of new protein and thus observe degradation of TRAF3 by Western blot. RANKL accelerated the degradation of TRAF3, starting at 1 hour and continuing through 4 hours (Figure 6B). In contrast, TNF prevented TRAF3 degradation, suggesting that TNF induction of TRAF3 results in the accumulation of NF-кB p100 and inhibition of osteoclastogenesis. To test this possibility, WT OCPs were transfected with TRAF3 siRNA to examine its effects on expression of NIK and p100 and on osteoclastogenesis. Transfection of TRAF3 siRNA reduced TRAF3 protein levels, and this was associated with higher levels of NIK and lower p100 levels in the TNF-treated cells compared with control siRNA-treated cells (Figure 6C). Surprisingly, we did not detect a significant change in p52 levels in either cytoplasmic or nuclear extracts using TRAF3 siRNA. This was a consistent finding that will require further study to determine whether TRAF3 also regulates p52 expression. TRAF3 siRNA significantly increased TNF-induced nuclear and cytoplasmic RelB, the typical partner of p52, which was reported recently to be involved in RANKL-induced osteoclastogenesis in vitro (44). Consistent with these results, TRAF3 siRNA significantly increased TNFinduced osteoclastogenesis (Figure 6D), although it had no effect on osteoclast numbers in RANKL-treated cultures, presumably because RANKL can degrade TRAF3 and TRAF3 siRNA likely would not have an additional effect on TRAF3 degradation. Of note, inhibition of TRAF3 attenuated the TNF-induced reduction of RANKL-mediated osteoclastogenesis. 


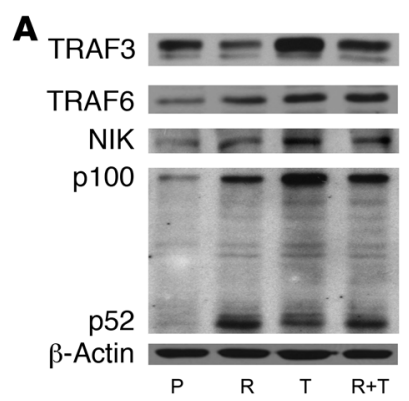

B
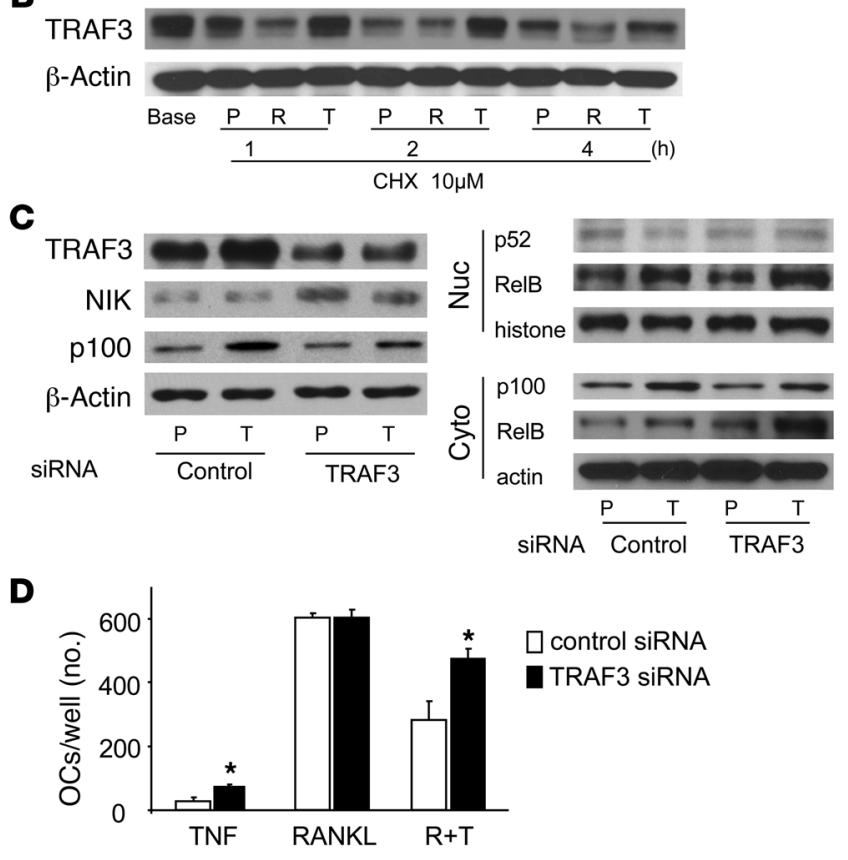

\section{Discussion}

There is compelling evidence for a strong proresorptive function of TNF, which has long been implicated in the pathogenesis of bone loss and inflammation in a variety of common bone diseases $(1,4,10,45)$ and, more recently, cherubism (46). TNF induces bone loss by indirect and direct mechanisms, including promotion of RANKL expression by accessory cells $(4,6)$ and induction of OCP proliferation and differentiation (47). These cells can then secrete proinflammatory osteoclastogenic cytokines and thus lead to the initiation of autocrine and paracrine self-amplifying cycles that increase bone loss (48). A pivotal role of TNF in pathologic bone loss is evidenced by the efficacy of anti-TNF therapy to limit disease progression in approximately $70 \%$ of patients with RA (49) and to reduce bone erosion soon after menopause (2).

Despite this evidence, TNF does not induce osteoclast formation when administered in vivo to $\mathrm{Rankl}^{-/-}$or $\mathrm{Rank}^{-/-}$mice (34), although it can induce osteoclastogenesis directly from Rank $^{-/-}$ OCPs in vitro when costimulated with TGF- $\beta$ (18), leading some to suggest that prior priming of OCPs by RANKL (35) or IL-1 expression by stromal cells (50) is necessary for TNF-induced bone resorption. Here we explain these discrepant findings by showing that locally injected TNF fails to induce osteoclastogenesis in $\mathrm{Rankl}^{-/}$and Rank $\mathrm{R}^{-/}$mice because of the inhibitory effects NF-KB p100 and provide conclusive evidence that TNF

\section{Figure 6}

TNF-induced TRAF3 negatively regulates osteoclastogenesis through NIK. (A) WT mouse OCPs were treated with RANKL and/or TNF for 8 hours, and whole-cell lysate protein was extracted and subjected to Western blotting for TRAF3, TRAF6, NIK, and NF-кB p100 and p52. (B) Cycloheximide $(10 \mu \mathrm{M})$ was added to WT mouse OCPs treated with RANKL (10 ng/ml), TNF $(20 \mathrm{ng} / \mathrm{ml})$, or PBS control for the indicated times. Whole-cell lysates were subjected to Western blotting for TRAF3. (C) WT mouse OCPs were transfected with TRAF3 siRNA or a nonspecific control siRNA for 8 hours. The cells were treated with TNF $(20 \mathrm{ng} / \mathrm{ml})$ or PBS for an additional 8 hours. Whole-cell lysates were subjected to Western blotting to determine the levels of cytoplasmic TRAF3, NIK, and NF-KB p100 (left panel) and either nuclear p52 or cytoplasmic p100 and RelB (right panels). (D) TRAF3 siRNA-transfected cells were treated with TNF and/or RANKL $(10 \mathrm{ng} / \mathrm{ml})$ for 3 days in the presence of M-CSF to generate osteoclasts. ${ }^{*} P<0.05$ vs. control).

can induce osteoclastogenesis in these mice when they are deficient also in NF- $\mathrm{KB}$ p100.

The osteoclastogenesis induced locally by TNF in calvariae of $\mathrm{Rank}^{-/-} / \mathrm{Nfkb2}^{-/-}$or Rankl $\mathrm{H}^{-/} / \mathrm{Nfkb2}^{-/-}$mice was associated with systemic induction of small numbers of mainly mononuclear $\mathrm{TRAP}^{+}$cells along epiphyseal growth plates. We have reported that RANKL induced by BMP2 in hypertrophic chondrocytes at the growth plate attracts OCPs to this site to remove bone (51), which is formed rapidly and must be removed to prevent development of osteopetrosis during development. Our findings suggest that the administered TNF directed OCPs to this site independently of RANKL. However, further studies are required to determine whether this was by a direct or indirect mechanism and whether TNF induces expression of chemokines here as it also does to attract circulating OCPs to inflamed joints (52).

Our observation that $\mathrm{TRAP}^{+}$cells do not form in untreated Rank $^{-/ /} / \mathrm{Nfkb}^{-/-}$or Rankl-//Nfkb2-/- mice is important because it suggests that TNF levels at the growth plate during endochondral ossification are too low to induce OCP differentiation to osteoclasts and that TNF does not have an important positive or negative regulatory role in this physiologic process. However, TRAP5b released from osteoclasts induced by locally injected TNF resulted in slightly increased serum TRAP5b levels in $\mathrm{Rank}^{-/ /} / \mathrm{Nfkb}^{+/-}$mice and significantly increased levels in Rank ${ }^{-/} / \mathrm{Nfkb}^{-/-}$mice, supporting our conclusion that TNF-induced resorption in pathologic bone remodeling is attenuated by NF- $\mathrm{\kappa B} 2$. The inhibitory role for NF-KB p100 in osteoclastogenesis is further supported by the development of erosive arthritis and systemic bone loss in TNF-Tg/

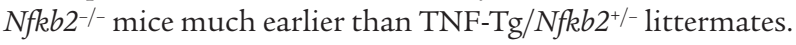

The reports that TNF induces RANKL expression in the joints of TNF-Tg mice $(53,54)$ led us to consider whether NF-KB p100 also limits RANKL-induced resorption in TNF-Tg mice. Indeed, we found that TNF limits RANKL-induced differentiation of WT OCPs in vitro through induced NF-KB p100 and that retroviral expression of NF-KB2 in OCPs reduced RANKL-induced osteoclastogenesis. Interestingly, inhibition of osteoclastogenesis alone does not prevent TNF-induced synovial inflammation, since TNF-Tg/ c-Fos ${ }^{-/}$hybrid mice lack osteoclasts and joint destruction but still have synovial inflammation (55). Therefore, inhibition of osteoclasts alone is likely to have a limited role in the treatment of RA. Unexpectedly and importantly, we found that the TNF-Tg/Nfkb2-/mice also had significantly increased inflammation in their joints, indicating that NF- $\mathrm{KB}$ p100 limits not only OCP differentiation 
but also the number of inflammatory cells attracted to the joints of the mice in response to TNF.

Deficiency of Nfkb2 dramatically accelerated TNF-Tg-induced arthritic bone erosion and inflammation, but this was not associated with an increase in their serum concentrations of either human or murine TNF, suggesting that deficiency of NF-кB p100 could be associated with more severe joint inflammation in RA patients. This might be of great importance in the clinical setting of arthritis, but it will require further study to determine whether there are variations in the transcription, function, or degradation of p100 that could increase the susceptibility of RA patients to TNF. Similarly, further study is also required to determine the precise mechanism whereby 100 limits the inflammatory infiltration, and specifically whether NF-KB p100 can be further increased locally, for example by using local adenoviral gene delivery, to inhibit inflammation and bone resorption in these mice.

TNF stimulates $N f k b 2$ mRNA expression through the canonical

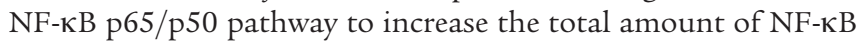
p100 transcripts and protein (24). At the same time, TNF activates the noncanonical pathway leading to some processing of NF- $\kappa B$ p100 to NF-кB p52 (24), but the molecular mechanisms have not been identified. We found that TNF stabilized TRAF3, resulting in its accumulation in OCPs in parallel with p100 levels, a finding that, to our knowledge, has not been reported previously in any cell type.

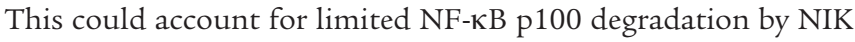
because TRAF3 induces degradation of NIK in B cells $(26,27)$. These effects of TNF differ from those of RANKL, which does not increase TRAF3 protein levels and activates NIK to induce efficient processing of p100 to p52 (23). TRAF3 negatively regulates $\mathrm{p} 100$ processing to $\mathrm{p} 52$ by promoting proteasomal degradation of NIK through its physical association with the TRAF3 binding motif in NIK (25, 26). Loss of TRAF3 results in accumulation of NIK and constitutive p100 processing in TRAF3 $^{-/-}$B cells $(25,28)$. TRAF6, which mediates RANKL/RANK-activated canonical NF- $\kappa B$ signaling, cannot associate with NIK directly and therefore does not inhibit it (56).

TRAF3 siRNA reduced TRAF3 protein levels in WT OCPs associated with increased NIK levels and p100 processing, which released more RelB to go to nuclei in the cells and increased osteoclastogenesis. TNF induced only a slight increase NIK levels in the OCPs, but this in part may reflect the difficulty in detecting low levels of NIK in cells with currently available reagents (57) and the fact that most published studies were able to detect changes in NIK levels only when NIK was overexpressed $(26,57)$. Although some investigators have suggested that TRAF2 is required for TNF-induced osteoclastogenesis (14) and TRAF5 is involved in both RANKLand TNF-induced osteoclastogenesis (15), others have found that TRAF2- or TRAF5-deficient OCPs can differentiate into mature osteoclasts in response to TNF (18). TRAF2 functions along with TRAF3 in B cells to degrade NIK (26). However, TRAF2, -5 , and -6 do not appear to play a major role in p100 processing in OCPs, based on our observation that their protein levels are similar in TNF- and RANKL-treated cells. Currently, we do not know the precise molecular mechanism by which TNF increases TRAF3 protein levels in OCPs. TNF does not affect TRAF3 mRNA, but it clearly prevents its degradation. It will be important to work this out in further studies, since stabilization of TRAF3 could potentially be one mechanism to limit bone resorption and possibly inflammation in inflammatory arthritis.

Our findings, coupled with the fact that TNF, RANKL, and IL-1 can all induce TNF expression by OCPs (40), support an impor- tant direct role for TNF in osteoclastogenesis in conditions such as RA and postmenopausal osteoporosis, in which production of these cytokines is increased beyond physiologic levels (58), while physiologic levels of the cytokines are unable to induce osteoclastogenesis in $\mathrm{Rankl}^{-/-}$and $\mathrm{Rank}^{-/-}$mice. However, they also identify an important negative regulatory role for TNF to limit its effects and those of RANKL. Given the important role that TNF has in inducing inflammation and bone destruction in many common bone diseases, we propose that this negative regulatory role for TNF in limiting bone resorption and inflammation might be harnessed to help reduce the high morbidity associated with many common diseases in which its expression is increased. These might include increasing the stability or expression of p100 or TRAF3 and inhibiting NIK in OCPs.

\section{Methods}

Reagents and animals. Recombinant M-CSF, RANKL, and TNF for cell cultures were from R\&D Systems. TNF for in vivo experiments was a gift from Amgen. Cycloheximide was from Calbiochem. The sources of $\mathrm{Nfkb2^{-/- }}$ $(\mathrm{C} 57 \mathrm{BL} / 6 \times 129)(11,31)$, human TNF-Tg $(\mathrm{CBA} \times \mathrm{C} 57 \mathrm{BL} / 6, \mathrm{TNF}-\mathrm{Tg}$ line 3647) (40), Rankl - $^{-}$(C57BL/6) (59), and Rank $k^{-/}$(C57BL/6) mice (30) were described previously. $\mathrm{Nkb2}^{-/-}$mice were crossed with $\mathrm{Rank}^{+/-}$or $\mathrm{Rankl}^{+/-}$

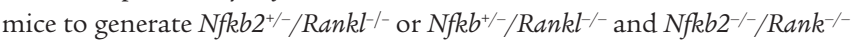
or Nfkb2-/-/Rank $k^{-/-}$mice. $N f k b 2^{-/-}$mice were crossed with TNF-Tg mice to

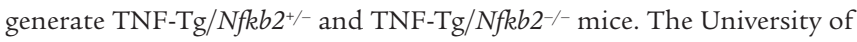
Rochester Medical Center Institutional Animal Care and Use Committee approved all animal studies.

Osteoclast formation and functional assays. Spleen cells $\left(1.75 \times 10^{5} /\right.$ well in 96-well plates) from WT mice were cultured with M-CSF $(10 \mathrm{ng} / \mathrm{ml})$ for $2-3$ days in $\alpha$-MEM with $10 \%$ FBS to enrich for OCPs. To generate osteoclasts from Rankl $l_{--}$and Rank $^{-/-}$mice in response to TNF, we seeded about $1 / 5$ th the number of splenocytes we used from WT cells. This allowed us to obtain comparable numbers of OCPs after 3-day treatment with $\mathrm{M}$-CSF and to induce osteoclastogenesis from these splenocytes. OCPs were treated with RANKL (10 ng/ml) and/or TNF (20 ng/ml) for 2-3 days to generate mature osteoclasts. To test the role of NF-KB p100 on OCP

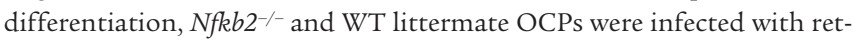
roviral supernatants of $\mathrm{p} 100$, p52, or GFP controls for 2 days and then treated with TNF or RANKL for 2-4 days, as described previously (11, 40). To test the role of TRAF3 in TNF-induced osteoclastogenesis, WT OCPs were transfected with a pool of 3 TRAF3-specific siRNAs (Santa Cruz Biotechnology Inc.) or nonspecific control siRNA for 8 hours, and then treated with TNF and/or RANKL for 3-4 days. For functional assays of osteoclasts, spleen cells were seeded in 96-well plates containing bovine cortical bone slices and cultured with TNF or RANKL, and resorption pit formation was assessed, as reported previously $(11,40)$. Cells were costimulated with M-CSF in all experiments.

Western blot analysis. Whole-cell lysate protein from OCPs or mature osteoclasts and nuclear protein from TRAF3 siRNA-transfected OCPs cultured in 60 - or $100-\mathrm{mm}$ dishes were prepared as described previously (11). Lysates $(10 \mu \mathrm{g})$ were loaded in 10\% SDS-PAGE gels and immunoblotted with antibodies to NF-кB1, NF-кB2, p65, RelB, TRAF2, TRAF3, TRAF5, TRAF6, and NIK (Santa Cruz Biotechnology Inc.) or mouse actin (SigmaAldrich). To test for degradation of the TRAF3 protein, $10 \mu \mathrm{M}$ CHX was added to WT OCPs treated with RANKL (10 ng/ml), TNF $(20 \mathrm{ng} / \mathrm{ml})$, or PBS control to inhibit new protein synthesis, and whole-cell lysates were subjected to Western blot for TRAF3.

TNF-induced osteoclastogenesis in vivo. TNF $(0.5 \mu \mathrm{g})$ or PBS was injected over the calvariae of 3- to 4-week-old mice twice daily for 5 days. Mice were sacrificed on day 6 , and calvariae and hind limbs were fixed in $10 \%$ formalin 
and decalcified with $10 \%$ EDTA. TRAP activity was assessed in paraffinembedded sections, as described previously $(11,40)$. Osteoclast numbers and surfaces and eroded surfaces were measured using an Osteometrics system, as described previously $(11,40)$.

Evaluation of arthritis and osteoporosis. Although the features of arthritis in the commonly used 197 line of TNF-Tg mice include paw swelling, reduced strength, and deformation (60), we found that the only easily identifiable, reproducible parameter in our 3647 line of TNF-Tg mice, which carry only 1 copy of the TNF transgene (40), is paw and finger deformation, including atrophy. We generated a deformation score ( 0 , no deformation; 1 , mild deformation; 2, moderate deformation; 3 , severe deformation; 4 , very severe deformation), which was evaluated once each week to determine the clinical progress of arthritis. Each forepaw and hindpaw was evaluated separately, and the deformation score was calculated as the sum of the 4 paws. Mice were sacrificed in $\mathrm{aCO}_{2}$ container. Right tibiae were collected for $\mu \mathrm{CT}$ scanning to evaluate cortical and trabecular bone volume. Left tibiae and forepaws were fixed, decalcified, and processed through paraffin for histologic evaluation of osteoclasts and arthritis, including inflammatory tissue area and carpal bone eroded cartilage surface.

ELISA. Mouse TRAP5b (Immunodiagnostic Systems) were assessed in

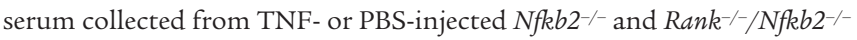

mice and their littermate controls, and human and mouse TNF (eBioscience) were determined in serum of TNF-Tg/ $\mathrm{Nfkb2}^{-/-}$mice and their littermate controls according to the manufacturer's instructions

Statistics. All results are given as mean \pm SD. Comparisons between 2 groups were analyzed using the 2-tailed unpaired Student's $t$ test. Oneway ANOVA and Dunnett's post-hoc multiple comparisons were used for comparisons among 3 or more groups. $P$ values less than 0.05 were considered statistically significant.

\section{Acknowledgments}

We thank Hani A. Awad for help with $\mu \mathrm{CT}$ analysis and Xiaoyun Zhang and Yanyun Li for histology. This work was supported by NIH grants AR43510 to B.F. Boyce and AR48697 to L. Xing.

Received for publication January 26, 2009, and accepted in revised form August 19, 2009.

Address correspondence to: Brendan F. Boyce, Department of Pathology and Laboratory Medicine, 601 Elmwood Ave., Box 626, Rochester, New York 14642, USA. Phone: (585) 275-5837; Fax: (585) 273-3637; E-mail: Brendan_Boyce@urmc.rochester.edu.
1. Novack, D.V., and Teitelbaum, S.L. 2008. The osteoclast: friend or foe? Annu. Rev. Pathol. 3:457-484.

2. Charatcharoenwitthaya, N., Khosla, S., Atkinson, E.J., McCready, L.K., and Riggs, B.L. 2007. Effect of blockade of TNF-alpha and interleukin-1 action on bone resorption in early postmenopausal women. J. Bone Miner. Res. 22:724-729.

3. Eghbali-Fatourechi, G., et al. 2003. Role of RANK ligand in mediating increased bone resorption in early postmenopausal women. J. Clin. Invest. 111:1221-1230.

4. Feng, X. 2005. Regulatory roles and molecular signaling of TNF family members in osteoclasts. Gene. 350:1-13.

5. Teitelbaum, S.L. 2000. Bone resorption by osteoclasts. Science. 289:1504-1508.

6. Boyce, B.F., and Xing, L. 2008. Functions of RANKL/ RANK/OPG in bone modeling and remodeling. Arch. Biochem. Biophys. 473:139-146.

7. Dempsey, P.W., Doyle, S.E., He, J.Q., and Cheng, G. 2003. The signaling adaptors and pathways activated by TNF superfamily. Cytokine Growth Factor Rev. 14:193-209.

8. Darnay, B.G., Besse, A., Poblenz, A.T., Lamothe, B., and Jacoby, J.J. 2007. TRAFs in RANK signaling. Adv. Exp. Med. Biol. 597:152-159.

9. Kim, H.H., et al. 1999. Receptor activator of NFkappaB recruits multiple TRAF family adaptors and activates c-Jun N-terminal kinase. FEBS Lett. 443:297-302.

10. Takayanagi, H. 2007. Osteoimmunology: shared mechanisms and crosstalk between the immune and bone systems. Nat. Rev. Immunol. 7:292-304.

11. Yamashita, T., et al. 2007. NF-kappaB p50 and p52 regulate receptor activator of NF-kappaB ligand (RANKL) and tumor necrosis factor-induced osteoclast precursor differentiation by activating c-Fos and NFATc1. J. Biol. Chem. 282:18245-18253.

12. Lomaga, M.A., et al. 1999. TRAF6 deficiency results in osteopetrosis and defective interleukin-1, CD40, and LPS signaling. Genes Dev. 13:1015-1024.

13. Naito, A., et al. 1999. Severe osteopetrosis, defective interleukin-1 signalling and lymph node organogenesis in TRAF6-deficient mice. Genes Cells. 4:353-362.

14. Kanazawa, K., and Kudo, A. 2005. TRAF2 is essential for TNF-alpha-induced osteoclastogenesis. J. Bone Miner. Res. 20:840-847.

15. Kanazawa, K., Azuma, Y., Nakano, H., and Kudo, A. 2003. TRAF5 functions in both RANKL- and TNFalpha-induced osteoclastogenesis. J. Bone
Miner. Res. 18:443-450.

16. Ross, F.P. 2006. M-CSF, c-Fms, and signaling in osteoclasts and their precursors. Ann. N. Y. Acad. Sci. 1068:110-116.

17. Kobayashi, K., et al. 2000. Tumor necrosis factor alpha stimulates osteoclast differentiation by a mechanism independent of the ODF/RANKLRANK interaction. J. Exp. Med. 191:275-286.

18. Kim, N., et al. 2005. Osteoclast differentiation independent of the TRANCE-RANK-TRAF6 axis. J. Exp. Med. 202:589-595.

19. Hayden, M.S., and Ghosh, S. 2004. Signaling to NF-kappaB. Genes Dev. 18:2195-2224.

20. Beinke, S., and Ley, S.C. 2004. Functions of NF-kappaB1 and NF-kappaB2 in immune cell biology. Biochem. J. 382:393-409.

21. Coux, O., and Goldberg, A.L. 1998. Enzymes catalyzing ubiquitination and proteolytic processing of the p105 precursor of nuclear factor kappaB1. J. Biol. Chem. 273:8820-8828.

22. Yilmaz, Z.B., Weih, D.S., Sivakumar, V., and Weih, F. 2003. RelB is required for Peyer's patch development: differential regulation of p52-RelB by lymphotoxin and TNF. EMBO J. 22:121-130.

23. Novack, D.V., et al. 2003. The IkappaB function of NF-kappaB2 p100 controls stimulated osteoclastogenesis. J. Exp. Med. 198:771-781.

24. Chaisson, M.L., et al. 2004. Osteoclast differentiation is impaired in the absence of inhibitor of kappa B kinase alpha. J. Biol. Chem. 279:54841-54848.

25. Zarnegar, B., Yamazaki, S., He, J.Q., and Cheng, G. 2008. Control of canonical NF-kappaB activation through the NIK-IKK complex pathway. Proc. Natl. Acad. Sci. U. S. A. 105:3503-3508.

26. Vallabhapurapu, S., et al. 2008. Nonredundant and complementary functions of TRAF2 and TRAF3 in a ubiquitination cascade that activates NIK-dependent alternative NF-kappaB signaling. Nat. Immunol. 9:1364-1370.

27. Hauer, J., et al. 2005. TNF receptor (TNFR)-associated factor (TRAF) 3 serves as an inhibitor of TRAF2/5-mediated activation of the noncanonical NF-kappaB pathway by TRAF-binding TNFRs. Proc. Natl. Acad. Sci. U. S. A. 102:2874-2879.

28. He, J.Q., et al. 2006. Rescue of TRAF3-null mice by p100 NF-kappa B deficiency. J. Exp. Med. 203:2413-2418.

29. Lacey, D.L., et al. 1998. Osteoprotegerin ligand is a cytokine that regulates osteoclast differentiation and activation. Cell. 93:165-176.
30. Dougall, W.C., et al. 1999. RANK is essential for osteoclast and lymph node development. Genes Dev. 13:2412-2424.

31. Franzoso, G., et al. 1997. Requirement for NF-kap$\mathrm{paB}$ in osteoclast and B-cell development. Genes Dev. 11:3482-3496.

32. Iotsova, V., Caamano, J., Loy, J., Lewin, A., and Bravo, R. 1997. Osteopetrosis in mice lacking NF-кB1 and NF-кB2. Nat. Med. 3:1285-1289.

33. Abu-Amer, Y., et al. 2000. Tumor necrosis factor receptors types 1 and 2 differentially regulate osteoclastogenesis. J. Biol. Chem. 275:27307-27310.

34. Li, J., et al. 2000. RANK is the intrinsic hematopoietic cell surface receptor that controls osteoclastogenesis and regulation of bone mass and calcium metabolism. Proc. Natl. Acad. Sci. U. S. A. 97:1566-1571.

35. Lam, J., et al. 2000. TNF-alpha induces osteoclastogenesis by direct stimulation of macrophages exposed to permissive levels of RANK ligand. J. Clin. Invest. 106:1481-1488.

36. Derudder, E., et al. 2003. RelB/p50 dimers are differentially regulated by tumor necrosis factor-alpha and lymphotoxin-beta receptor activation: critical roles for p100. J. Biol. Chem. 278:23278-23284.

37. Takayanagi, H., et al. 2002. RANKL maintains bone homeostasis through c-Fos-dependent induction of interferon-beta.[see comment]. Nature. 416:744-749.

38. Boyle, W.J., Simonet, W.S., and Lacey, D.L. 2003. Osteoclast differentiation and activation. Nature. 423:337-342.

39. Xing, L., et al. 2003. Expression of either NF-kap$\mathrm{paB}$ p50 or p52 in osteoclast precursors is required for IL-1-induced bone resorption. J. Bone Miner. Res. 18:260-269.

40. Yao, Z., Xing, L., Qin, C., Schwarz, E.M., and Boyce, B.F. 2008. Osteoclast precursor interaction with bone matrix induces osteoclast formation directly by an interleukin-1-mediated autocrine mechanism. J. Biol. Chem. 283:9917-9924.

41. Basak, S., et al. 2007. A fourth IkappaB protein within the NF-kappaB signaling module. Cell. 128:369-381.

42. Li, P., et al. 2004. RANK signaling is not required for TNFalpha-mediated increase in CD11(hi) osteoclast precursors but is essential for mature osteoclast formation in TNFalpha-mediated inflammatory arthritis. J. Bone Miner. Res. 19:207-213.

43. Ochi, S., et al. 2007. Pathological role of osteoclast costimulation in arthritis-induced bone loss. Proc. 
Natl. Acad. Sci. U. S. A. 104:11394-11399.

44. Vaira, S., et al. 2008. RelB is the NF-kappaB subunit downstream of NIK responsible for osteoclast differentiation. Proc. Natl. Acad. Sci. U. S. A 105:3897-3902.

45. Boyce, B.F., et al. 2005. TNF-alpha and pathologic bone resorption. Keio J. Med. 54:127-131.

46. Aliprantis, A.O., et al. 2008. NFATc1 in mice represses osteoprotegerin during osteoclastogenesis and dissociates systemic osteopenia from inflammation in cherubism. J. Clin. Invest. 118:3775-3789.

47. Yao, Z., et al. 2006. Tumor necrosis factor-\{alpha\} increases circulating osteoclast precursor numbers by promoting their proliferation and differentiation in the bone marrow through up-regulation of c-Fms expression. J. Biol. Chem. 281:11846-11855.

48. Boyce, B.F., Schwarz, E.M., and Xing, L. 2006 Osteoclast precursors: cytokine-stimulated immunomodulators of inflammatory bone disease. Curr. Opin. Rheumatol. 18:427-432.

49. Shealy, D.J., and Visvanathan, S. 2008. Anti-TNF antibodies: lessons from the past, roadmap for the future. Handb. Exp. Pharmacol. 181:101-129.

50. Wei, S., Kitaura, H., Zhou, P., Ross, F.P., and Teitelbaum, S.L. 2005. IL-1 mediates TNF-induced osteoclastogenesis. J. Clin. Invest. 115:282-290.

51. Usui, M., et al. 2008. Murine and chicken chondrocytes regulate osteoclastogenesis by producing RANKL in response to BMP2. J. Bone Miner. Res. 23:314-325.

52. Xing, L., Schwarz, E.M., and Boyce, B.F. 2005. Osteoclast precursors, RANKL/RANK, and immunology. Immunol. Rev. 208:19-29.

53. Zhang, Q., et al. 2008. VEGF-C, a lymphatic growth factor, is a RANKL target gene in osteoclasts that enhances osteoclastic bone resorption through an autocrine mechanism. J. Biol. Chem. 283:13491-13499.

54. Schwarz, E.M., et al. 2006. Autoimmunity and bone. Ann. N. Y. Acad. Sci. 1068:275-283.

55. Redlich, K., et al. 2002. Osteoclasts are essential for TNF-alpha-mediated joint destruction. J. Clin. Invest. 110:1419-1427.

56. He, J.Q., Saha, S.K., Kang, J.R., Zarnegar, B., and
Cheng, G. 2007. Specificity of TRAF3 in its negative regulation of the noncanonical NF-kappa B pathway. J. Biol. Chem. 282:3688-3694.

57. Sasaki, Y., et al. 2008. NIK overexpression amplifies, whereas ablation of its TRAF3-binding domain replaces BAFF:BAFF-R-mediated survival signals in B cells. Proc. Natl. Acad. Sci. U. S. A. 105:10883-10888.

58. O'Gradaigh, D., Ireland, D., Bord, S., and Compston, J.E. 2004. Joint erosion in rheumatoid arthritis: interactions between tumour necrosis factor alpha, interleukin 1 , and receptor activator of nuclear factor kappaB ligand (RANKL) regulate osteoclasts. Ann. Rheum. Dis. 63:354-359.

59. Kim, N., Odgren, P.R., Kim, D.K., Marks, S.C., Jr., and Choi, Y. 2000. Diverse roles of the tumor necrosis factor family member TRANCE in skeletal physiology revealed by TRANCE deficiency and partial rescue by a lymphocyte-expressed TRANCE transgene. Proc. Natl. Acad. Sci. U. S. A. 97:10905-10910.

60. Keffer, J., et al. 1991. Transgenic mice expressing human tumour necrosis factor: a predictive genetic model of arthritis. EMBOJ. 10:4025-4031. 